\title{
Incidence and risk factors of surgical site infection following colorectal surgery in China: a national cross-sectional study
}

Xufei Zhang ${ }^{1}$, Zhiwei Wang ${ }^{2}$, Jun Chen ${ }^{3}$, Peige Wang ${ }^{4}$, Suming Luo ${ }^{5}$, Xinjian Xu' ${ }^{6}$, Wei Mai ${ }^{7}$, Guangyi Li ${ }^{8}$, Gefei Wang ${ }^{3}$, Xiuwen $\mathrm{Wu}^{3}$ and Jianan Ren ${ }^{1,3^{*}}$ (D)

\begin{abstract}
Purposes: Surgical site infection (SSI) after colorectal surgery is a frequent complication associated with the increase in morbidity, medical expenses, and mortality. To date, there is no nationwide large-scale database of SSI after colorectal surgery in China. The aim of this study was to determine the incidence of SSI after colorectal surgery in China and to further evaluate the related risk factors.

Methods: Two multicenter, prospective, cross-sectional studies covering 55 hospitals in China and enrolling adult patients undergoing colorectal surgery were conducted from May 1 to June 30 of 2018 and the same time of 2019. The demographic and perioperative characteristics were collected, and the main outcome was SSI within postoperative 30 days. Multivariable logistic regressions were conducted to predict risk factors of SSI after colorectal surgery.

Results: In total, 1046 patients were enrolled and SSI occurred in 74 patients (7.1\%). In the multivariate analysis with adjustments, significant factors associated with SSI were the prior diagnosis of hypertension (OR, 1.903; 95\% confidence interval $[C l], 1.088-3.327, P=0.025)$, national nosocomial infection surveillance risk index score of 2 or $3(\mathrm{OR}, 3.840 ; 95 \% \mathrm{Cl}, 1.926-7.658, P<0.001)$, laparoscopic or robotic surgery (OR, 0.363 ; $95 \% \mathrm{Cl}, 0.200-0.659, P<0.001)$, and adhesive incise drapes $(\mathrm{OR}, 0.400 ; 95 \% \mathrm{Cl}, 0.187-0.855, P=0.018)$. In addition, SSI group had remarkably increased length of postoperative stays (median, $15.0 \mathrm{~d}$ versus 9.0d, $P<0.001$ ), medical expenses (median, 74,620 yuan versus 57,827 yuan, $P<0.001$ ), and the mortality $(4.1 \%$ versus $0.3 \%, P=0.006$ ), compared with those of non-SSI group.

Conclusion: This study provides the newest data of SSI after colorectal surgery in China and finds some predictors of SSI. The data presented in our study can be a tool to develop optimal preventive measures and improve surgical quality in China.
\end{abstract}

Keywords: SSI, Colorectal surgery, Prevalence, Risk factors, Multicenter study

\footnotetext{
* Correspondence: jiananr@gmail.com

${ }^{1}$ Research Institute of General Surgery, Jinling Hospital, Nanjing Medical University, Nanjing 210002, People's Republic of China

${ }^{3}$ Research Institute of General Surgery, Jinling Hospital, Medical School of Nanjing University, Nanjing 210002, People's Republic of China

Full list of author information is available at the end of the article
} changes were made. The images or other third party material in this article are included in the article's Creative Commons licence, unless indicated otherwise in a credit line to the material. If material is not included in the article's Creative Commons licence and your intended use is not permitted by statutory regulation or exceeds the permitted use, you will need to obtain permission directly from the copyright holder. To view a copy of this licence, visit http://creativecommons.org/licenses/by/4.0/ The Creative Commons Public Domain Dedication waiver (http://creativecommons.org/publicdomain/zero/1.0/) applies to the data made available in this article, unless otherwise stated in a credit line to the data. 


\section{Introduction}

To date, surgical site infection (SSI) is still one of the most common types of health care-related infection, especially in middle-income and low-income countries [1]. Moreover, SSI is accompanied with the prolongation of hospital stays and the increase of related medical expenses [2]. In addition, the occurrence of SSI continues to be an important role in nosocomial morbidity and mortality [3]. Thus, the prevention of SSI remains a pressing concern.

Because of the existence of numerous microbes in the rectum and colon, the SSI incidence of colorectal surgery is usually higher than those observed in other types of surgeries [4, 5]. Little multicenter surveillance focusing on SSI after colorectal surgery has been carried out in China. In 2019, Liu et al. [6] reported SSI and its risk factor in radical resection of colon or rectal carcinoma, but patients were rolled from January 2015 to June 2016. At present, there is a lack of recent studies to reflect the current situation of SSI after colorectal surgery in China in recent years.

Our group has conducted two multicenter, prospective, cross-sectional studies enrolling adult patients ( $>18$ years) who received colorectal surgery from May 1 to June 30 of 2018 and the same time of 2019 in China. This nationwide data aims to determine the incidence rate and related risk factors of SSI after colorectal surgery.

\section{Patients and methods}

\section{Study design}

Two multicenter, prospective, cross-sectional studies were conducted from May 1 to June 30 of 2018 and the same time of 2019, and enrolled adult patients ( $>18$ years) who received colorectal surgery in the department of general surgery of 55 hospitals in China during these periods (Supplementary file 1). The follow-up period was defined as 30 days after surgery. The exclusion criteria were pregnancy, patients undergoing urological, transplantation or gynecological surgery. The ethics committees of all institutions involved in the study approved our study. All enrolled patients should provide written informed consent ahead of their participation in the study. For data collecting, we only included those patients who have agreed to use the information from their medical records for the purpose of scientific research.

\section{Data collection}

Aside from baseline variables, we collected other data which may be related to the likelihood of SSI occurrence. Firstly, each hospital identified patients who met the inclusion criteria and could comply with follow-up, and collected their essential data. Secondly, we had specialized team members to evaluate the whole data collection process and the accuracy of all data.
Baseline variables of enrolled patients covered gender, age, body mass index (BMI), American Society of Anesthesiologists (ASA) physical status score, diagnosis, the prior diagnosis of diabetes mellitus, hypertension, chronic renal dysfunction (renal failure or dialysis), chronic hepatic dysfunction (abnormal concentrations of liver enzymes, hepatocellular carcinoma, cirrhosis or hepatitis), chronic cardiac dysfunction (heart failure, myocardial infarction, or previous cardiac surgery), tuberculosis, use of immunosuppressive medication, smoking status (nonsmoker, former smoker, or current smoker), preoperative blood biochemical parameters (albumin, hemoglobin and fasting blood glucose; collected at the preoperative day of surgery), and length of preoperative stay.

Patient perioperative characteristics covered type of surgery based on the urgency of surgery (emergency surgery or elective surgery), surgical site (colon or rectum), timing of hair removal (none, night before surgery, or day of surgery), surgical wound classes (dirty, cleancontaminated, or contaminated), ways of bowel preparation (oral antibiotics bowel preparation [OABP] without mechanical bowel preparation [MBP], MBP without OABP, or OABP combined with MBP), type of surgical hand preparation (scrubbing or disinfectant), surgery approach (open surgery, laparoscopy surgery or robotic surgery), incisional protection (adhesive incise drapes, wound edge protector, or gauze which is put around the incision to avoid the friction of surgical instruments on the incision skin and subcutaneous tissue), type of fluids for incisional wound irrigation (povidone-iodine solution, hydrogen peroxide solution, saline, or something else), fascial or muscle suture materials (silk suture, absorbable sutures, or antimicrobial-coated and absorbable sutures), skin closure materials (silk suture, absorbable sutures, skin staples, or something else), duration of surgery, grade of lead surgeon on the basis of their professional titles, colostomy/ileostomy, and the national nosocomial infections surveillance (NNIS) risk index.

The NNIS risk index is an internationally recognized method for stratifying surgical risk, while the NNIS risk index [7] varied from 0 to 3 by assessing three variables: duration of surgery, surgical wound class and ASA score. Each variable's cutoff values were a contaminated or dirty surgical incision, the operative duration of $225 \mathrm{~min}$ and an ASA score of 3 , with 1 point evaluated if any one variable was over the cutoff value.

In this study, the primary outcome was the occurrence of SSI within postoperative 30 days defined by Center for Disease Control criteria [8], including organ-space infections, deep or superficial incisional infections. The follow-up was carried out via standard telephone interview or review of the readmission records if patients were discharged from hospital after less than 
postoperative $30 \mathrm{~d}$. If patients had more than one type of SSI within 30 days, only a single form of SSI with the deepest anatomy was included in subsequent analysis. In addition, once SSI happened, the bacterial culture of secretion, the pus, pelvic puncture fluid, or distal catheter would be conducted, which depended on the criteria of each hospital.

The secondary outcomes were duration of total hospital stay and postoperative stay, costs, and postoperative 30-day mortality.

\section{Statistical analysis}

Results were shown as the mean \pm standard deviation (SD) or median with interquartile range (IQR), as appropriate. Comparisons of all continuous variables between the two groups were conducted by using the MannWhitney U-test or Student's t-test depending on Gaussian distribution. Comparisons of categorical variables were conducted using Fisher's exact test or chi-square test, as appropriate. The criterion of statistical significance was $P<0.05$.

Variables with statistical significance in univariable logistic regressions would be included into multivariable logistic regression analysis to identify the independent risk factors of SSI within postoperative 30 days.

We used SPSS v24 software to analyze all data.

\section{Results}

In total, 1046 patients receiving colorectal surgery were enrolled in this study, of which 74 patients developed SSI within postoperative 30 days, namely the incidence rate of SSI for 7.1\%. For patients with SSI, 24 developed organ-space infections, 18 developed deep incisional infections, and 32 developed superficial incisional infections. The demographics of the enrolled patients are exhibited in Table 1. There was no difference between non-SSI group and SSI group on gender, age, BMI, length of preoperative stay or smoking history. The most common comorbidities were hypertension and diabetes mellitus, and the two groups significantly differed in the presence or absence of hypertension $(P=0.006)$. Moreover, the incidence of SSI in patients with higher preoperative blood glucose was significantly increased $(P=$ $0.025)$. In addition, the overall rate of low albumin concentration $(<3.5 \mathrm{~g} / \mathrm{dl})$ in non-SSI group and SSI group was 20.5 and $43.2 \%(P<0.001)$.

The perioperative information of the patients is summarized in Table 2. Of note, the SSI incidence varied significantly based on surgical sites, of which colon surgery had higher SSI incidence (9.0\% versus $4.0 \%)$. The emergency surgery accounted for $6.5 \%$ of all enrolled patients. The clean-contaminated wound was the most common type of wound classes, and patients with an ASA score of 1 or 2 were over $70 \%$. The incidence of
SSI in patients with a clean-contaminated wound was significantly lower than those with a contaminated or dirty wound $(6.0 \%$ versus $25.9 \%, P<0.001)$. Regardless the timing of hair removal (the day of surgery or the night before surgery), patients undergoing hair removed had lower SSI incidence compared with those without hair removal $(P=0.028)$. In addition, the most common method of bowel preparation was MBP alone, and the incidence of SSI in patients with MBP alone was remarkably lower compared with those undergoing no bowel preparation $(P<0.001)$.

Regarding the approach of surgery, laparoscopic or robotic surgery had lower SSI incidence than open surgery (3.7\% versus $12.8 \%, P<0.001)$. Silk sutures were the most commonly used skin closure materials, but absorbable sutures had the lowest SSI incidence in comparison with other sutures $(P=0.027)$. There was no difference among patients receiving different fascial or muscle suture materials when comparing SSI. Among materials of incisional wound irrigation, saline was the most commonly used material. In patients with colostomy/ileostomy, 18.3\% developed SSI $(P<0.001)$. In addition, patients with the higher NNIS risk index scores had the remarkably higher incidence rate of SSI $(P<0.001)$.

The secondary outcomes of studies have been exhibited in Table 3. The median time of SSI occurrence is 5.5d (IQR: 3.0-9.0 d) after surgery. The SSI group had the remarkably longer lengths of total hospital stays compared with those in the non-SSI group $(22.5 \mathrm{~d}$ versus $15.0 \mathrm{~d}, P<0.001)$. Moreover, compared with the non-SSI group, there was the longer length of postoperative stays in the SSI group $(15.0 \mathrm{~d}$ versus $9.0 \mathrm{~d}, P<$ $0.001)$. The medical expenses of the non-SSI group were also lower compared with those of the SSI group $(P<0.001)$. During the study, a total of six patients died, and the mortality in the SSI group was also remarkably higher compared with that in the non-SSI group $(P=$ 0.006).

Table 4 shows the logistic regression analysis. The risk factors significantly associated with the occurrence of SSI were hypertension, tuberculosis, low albumin $(<3.5 \mathrm{~g} / \mathrm{dl})$, blood glucose $>150 \mathrm{mg} / \mathrm{dl}$, colon surgery, emergency surgery, colostomy/ileostomy and the higher NNIS risk index score (2 or 3). The protection factors of SSI were hair removal at the night before surgery, MBP alone, laparoscopic or robotic surgery and adhesive incise drapes. Furthermore, the multivariate analysis revealed that the independent risk factors of SSI following colorectal surgery were NNIS risk index score for 2 or 3 and hypertension, while incisional protection with adhesive incise drapes and laparoscopic or robotic surgery were protective factors for SSI. In three variables of NNIS risk index score, Supplementary file 2 revealed that the surgical wound class (contaminated or dirty) was were the main predictor of SSI. 
Table 1 Demographics of included patients

\begin{tabular}{|c|c|c|c|c|}
\hline Variables & Total & Non-SSI group & SSI group & $p$ value \\
\hline $\mathrm{N}(\%)$ & $1046(100 \%)$ & $972(92.9 \%)$ & $74(7.1 \%)$ & \\
\hline Age, years, median (IQR) & $59.0(52.0-70.0)$ & $62.0(53.0-70.0)$ & $60.5(50.0-71.0)$ & 0.841 \\
\hline Gender (\%) & & & & 0.302 \\
\hline Male & $619(59.2 \%)$ & $571(58.7 \%)$ & $48(64.9 \%)$ & \\
\hline Female & $427(40.8 \%)$ & $401(41.3 \%)$ & $26(35.1 \%)$ & \\
\hline BMI, median (IQR) & $23.0(20.3-25.4)$ & $23.0(20.3-25.2)$ & $23.9(19.9-26.3)$ & 0.421 \\
\hline \multicolumn{5}{|l|}{ Comorbidity (\%) } \\
\hline Diabetes mellitus & $128(12.2 \%)$ & $115(11.8 \%)$ & $13(17.6 \%)$ & 0.147 \\
\hline Hypertension & $257(24.6 \%)$ & $229(23.6 \%)$ & $28(37.8 \%)$ & 0.006 \\
\hline Chronic liver disease & $20(1.9 \%)$ & $20(2.1 \%)$ & $0(0 \%)$ & 0.390 \\
\hline Chronic kidney disease & $9(0.9 \%)$ & $9(0.9 \%)$ & $0(0 \%)$ & 1.00 \\
\hline Chronic heart disease & $40(3.8 \%)$ & $34(3.5 \%)$ & $6(8.1 \%)$ & 0.057 \\
\hline Tuberculosis & $5(0.5 \%)$ & $3(0.3 \%)$ & $2(2.7 \%)$ & 0.043 \\
\hline Steroid use & $9(0.9 \%)$ & $8(0.8 \%)$ & $1(1.4 \%)$ & 0.485 \\
\hline Immunosuppressive medication & $11(1.1 \%)$ & $9(0.9 \%)$ & $2(2.7 \%)$ & 0.180 \\
\hline Smoking history (\%) & & & & 0.980 \\
\hline No & $839(80.2 \%)$ & $780(80.2 \%)$ & 59 (79.7\%) & \\
\hline Former & $45(4.3 \%)$ & $42(4.3 \%)$ & $3(4.1 \%)$ & \\
\hline Current & $162(15.5 \%)$ & $150(15.4 \%)$ & $12(16.2 \%)$ & \\
\hline Hemoglobin, g/dl (\%) & & & & 0.183 \\
\hline$\geq 11$ & $762(72.8 \%)$ & $713(73.4 \%)$ & 49 (66.2\%) & \\
\hline$<11$ & $284(27.2 \%)$ & $259(26.6 \%)$ & $25(33.8 \%)$ & \\
\hline Albumin, g/dl (\%) & & & & $<0.001$ \\
\hline$\geq 3.5$ & 815 (77.9\%) & $773(79.5 \%)$ & $42(56.8 \%)$ & \\
\hline$<3.5$ & $231(22.1 \%)$ & $199(20.5 \%)$ & $32(43.2 \%)$ & \\
\hline Blood glucose, mg/dl (\%) & & & & 0.025 \\
\hline$<80$ & $181(17.3 \%)$ & $168(17.3 \%)$ & $13(17.6 \%)$ & \\
\hline $80 \sim 150$ & 771 (73.7\%) & $723(74.4 \%)$ & 48 (64.9\%) & \\
\hline$>150$ & $94(9.0 \%)$ & $81(8.3 \%)$ & $13(17.6 \%)$ & \\
\hline LPS, day, median (IQR) & $6(3.5-9.0)$ & $6(3-9)$ & 7.5 (4-9) & 0.130 \\
\hline
\end{tabular}

IQR interquartile range, $B M I$ body mass index, LPS Length of preoperative stay

\section{Discussion}

SSI is a common complication after colorectal surgery. Indeed, previous studies have reported that the incidence rate of SSI following colorectal surgery could be up to $20 \%$ [9-11]. Based on Japan nosocomial infection surveillance system national database from 2008 through 2010, the cumulative incidence rates of SSI for rectal and colon surgery were 15.0 and $17.8 \%$, respectively [12]. Additionally, the results of American College of Surgeons National Surgical Quality Improvement Program found a reduction in colorectal SSI incidence from $17.58 \%$ of 2011 to $5.11 \%$ of 2015 [13]. This comprehensive observational study investigated the SSI incidence and risk factors of colorectal surgery among 1046 patients from 55 hospitals in China. This study demonstrated that the incidence rate of SSI following colorectal surgery was $7.1 \%$. In addition, the occurrence of SSI remarkably increased the treatment expenses and mortality. The independent risk factor for SSI included hypertension and high NNIS risk index score, whereas using adhesive incise drapes to protect incisions and undergoing laparoscopic surgery or robotic surgery could effectively reduce the occurrence of SSI.

The present study identified that prior diagnosis of hypertension acted as an independent risk factor of SSI following colorectal surgery. Several studies have found hypertension is an important risk factor in spine operations [11], cesarean deliveries [14] and breast cancer surgery [15]. However, there is no specific study to discuss the association between SSI and hypertension after colorectal surgery. Intriguingly, it was reported that lowest 
Table 2 Perioperative characteristics of included patients

\begin{tabular}{|c|c|c|c|c|}
\hline Variables & Total $(n=1046)$ & Non-SSI group $(n=972)$ & SSI group $(n=74)$ & $p$ value \\
\hline Type of surgery (\%) & & & & 0.002 \\
\hline Colon surgery & $641(61.3 \%)$ & $583(60.0 \%)$ & $58(78.4 \%)$ & \\
\hline Rectal surgery & $405(38.7 \%)$ & $389(40.0 \%)$ & $16(21.6 \%)$ & \\
\hline Urgency of surgery (\%) & & & & $<0.001$ \\
\hline Elective & $978(93.5 \%)$ & $922(94.9 \%)$ & $56(75.7 \%)$ & \\
\hline Emergency & $68(6.5 \%)$ & $50(5.1 \%)$ & $18(24.3 \%)$ & \\
\hline ASA score (\%) & & & & 0.001 \\
\hline 1 or 2 & $818(78.2 \%)$ & $772(79.4 \%)$ & $46(62.2 \%)$ & \\
\hline 3 or 4 & $228(21.8 \%)$ & $200(20.6 \%)$ & $28(37.8 \%)$ & \\
\hline Surgical wound class (\%) & & & & $<0.001$ \\
\hline Clean-contaminated & $992(94.9 \%)$ & $932(95.9 \%)$ & $60(81.1 \%)$ & \\
\hline Contaminated or dirty & $54(5.2 \%)$ & $40(4.1 \%)$ & $14(18.9 \%)$ & \\
\hline Timing of hair removal (\%) & & & & 0.028 \\
\hline None & $188(18.0 \%)$ & $167(17.2 \%)$ & $21(28.4 \%)$ & \\
\hline Night before surgery & $672(64.2 \%)$ & $634(65.2 \%)$ & 38 (51.4\%) & \\
\hline Day of surgery & $186(17.8 \%)$ & $171(17.6 \%)$ & $15(20.3 \%)$ & \\
\hline Hair removal & & & & 0.016 \\
\hline No & $188(18.0 \%)$ & $167(17.2 \%)$ & $21(28.4 \%)$ & \\
\hline Yes & $858(82.0 \%)$ & $805(82.8 \%)$ & $53(71.6 \%)$ & \\
\hline Bowel preparation (\%) & & & & $<0.001$ \\
\hline None & $331(31.6 \%)$ & $294(30.2 \%)$ & 37 (50.0\%) & \\
\hline MBP only & $575(55.0 \%)$ & $549(56.5 \%)$ & $26(35.1 \%)$ & \\
\hline OABP only & $42(4.0 \%)$ & $36(3.7 \%)$ & $6(8.1 \%)$ & \\
\hline $\mathrm{MBP}+\mathrm{OABP}$ & 98 (9.4\%) & $93(9.6 \%)$ & $5(6.8 \%)$ & \\
\hline Hand preparation (\%) & & & & 0.209 \\
\hline Disinfectant & $831(79.4 \%)$ & $768(79.0 \%)$ & $63(85.1 \%)$ & \\
\hline Scrubbing & $215(20.6 \%)$ & $204(21.0 \%)$ & $11(14.9 \%)$ & \\
\hline Approach (\%) & & & & $<0.001$ \\
\hline Open & $391(37.4 \%)$ & $341(35.1 \%)$ & $50(67.6 \%)$ & \\
\hline Laparoscopic or robotic & $655(62.6 \%)$ & $631(64.9 \%)$ & $24(32.4 \%)$ & \\
\hline Incisional protection (\%) & & & & 0.151 \\
\hline None & $211(20.2 \%)$ & $191(19.7 \%)$ & $20(27.0 \%)$ & \\
\hline Gauze & $121(11.6 \%)$ & $113(11.6 \%)$ & $8(10.8 \%)$ & \\
\hline Adhesive incise drapes & $340(32.5 \%)$ & $324(33.3 \%)$ & $16(21.6 \%)$ & \\
\hline Wound edge protector & $374(35.8 \%)$ & $344(35.4 \%)$ & $30(40.5 \%)$ & \\
\hline Skin closure materials (\%) & & & & 0.027 \\
\hline Silk sutures & $627(60.0 \%)$ & $579(59.6 \%)$ & $48(64.9 \%)$ & \\
\hline Absorbable sutures & $264(25.2 \%)$ & $253(26.0 \%)$ & $11(14.9 \%)$ & \\
\hline Skin staples & $120(11.5 \%)$ & $111(11.4 \%)$ & $9(12.2 \%)$ & \\
\hline other & 35 (3.3\%) & $29(3.0 \%)$ & $6(8.1 \%)$ & \\
\hline Fascial or muscle suture materials (\%) & & & & 0.545 \\
\hline Silk sutures & $153(14.6 \%)$ & $140(14.4 \%)$ & $13(17.6 \%)$ & \\
\hline Absorbable sutures & $408(39.0 \%)$ & $377(38.8 \%)$ & $31(41.9 \%)$ & \\
\hline antimicrobial-coated and absorbable sutures & $485(46.4 \%)$ & $455(46.8 \%)$ & $30(40.5 \%)$ & \\
\hline
\end{tabular}


Table 2 Perioperative characteristics of included patients (Continued)

\begin{tabular}{|c|c|c|c|c|}
\hline Variables & Total $(n=1046)$ & Non-SSI group $(n=972)$ & SSI group $(n=74)$ & $p$ value \\
\hline Incisional wound irrigation (\%) & & & & 0.037 \\
\hline None & $81(7.7 \%)$ & $72(7.4 \%)$ & $9(12.2 \%)$ & \\
\hline Povidone-iodine solution & $182(17.4 \%)$ & $172(17.7 \%)$ & $10(13.5 \%)$ & \\
\hline Hydrogen peroxide solution & $51(4.9 \%)$ & $48(4.9 \%)$ & $3(4.1 \%)$ & \\
\hline Saline & $701(67.0 \%)$ & $655(67.4 \%)$ & $46(62.2 \%)$ & \\
\hline Other & $31(3.0 \%)$ & $25(2.6 \%)$ & $6(8.1 \%)$ & \\
\hline Colostomy/ileostomy & & & & $<0.001$ \\
\hline No & $920(88.0 \%)$ & $869(89.4 \%)$ & $51(68.9 \%)$ & \\
\hline Yes & $126(12.0 \%)$ & $103(10.6 \%)$ & $23(31.1 \%)$ & \\
\hline Grade of lead surgeon (\%) & & & & 0.068 \\
\hline Senior & $694(66.3 \%)$ & $651(67.0 \%)$ & $43(58.1 \%)$ & \\
\hline Middle & $304(29.1 \%)$ & $280(28.8 \%)$ & $24(32.4 \%)$ & \\
\hline Junior & $48(4.6 \%)$ & $41(4.2 \%)$ & $7(9.5 \%)$ & \\
\hline Surgical duration, min, median (IQR) & $180.0(130.0-225.0)$ & $180.0(130.0-220.0)$ & $193.5(143.8-254.3)$ & 0.019 \\
\hline NNIS risk index & & & & $<0.001$ \\
\hline 0 or 1 & $969(92.6 \%)$ & $916(94.2 \%)$ & $53(71.6 \%)$ & \\
\hline 2 or 3 & 77 (7.4\%) & $56(5.8 \%)$ & $21(28.4 \%)$ & \\
\hline
\end{tabular}

ASA American society of anesthesiologists physical status classification system, MBP mechanical bowel preparation, OABP oral antibiotic bowel preparation, NNIS national nosocomial infections surveillance

postoperative mean arterial pressure was related to surgical site infection following colorectal surgery, but postoperative time-weighted mean arterial pressure was not [16]. Although hypertension was found to be the major risk factor, some details including the variation of blood pressure during the perioperative period were not recorded in our study, which could be a focus in our future research.

We also showed that NNIS risk index score for 2 or 3 was an independent predictor of SSI, which is consistent with our previous study [17]. The NNIS risk index contains the wound class, the ASA score, and the duration of surgery. The present study demonstrated that the surgical wound class (contaminated or dirty) was the main predictor of SSI among three variables of NNIS risk index score. The odds ratio of the ASA score (level of 3 or 4) was second to that of the surgical wound class (contaminated or dirty). The ASA score (level of 4 and 5) has been reported to be an important risk factor for SSI [18]. Therefore, patients with higher ASA score may be inclined to suffer more risks of SSI after surgery.

As is well-known, laparoscopic surgery and robotic surgery are becoming cumulatively common worldwide. Several studies have found that laparoscopic approach remarkably reduces SSI following colorectal surgery [19, 20]. Our study enrolled 50 patients receiving robotic surgery, two of which had SSI following surgery. The incidence rate of SSI in patients undergoing robotic surgery was $4.0 \%$, which was similar to that undergoing laparoscopic surgery (3.6\%) and was much lower than open surgery (12.8\%). Thus, we combined patients accepting either robotic surgery or laparoscopic surgery into the same group, since both of them could decrease the SSI incidence by contrast with open surgery.

To better strengthen the aspects associated with wound edge isolation, devices of incisional protection have been manufactured and marketed. In this study, we found that there were three main kinds of incisional protection devices, comprising wound edge protectors, adhesive incise drapes and gauze, used for colorectal surgery in China. Although both single- and double-ring wound edge protectors have been observed to be the beneficial effect to reduce the SSI rate in previous studies [21-23], only adhesive incise drapes could be the independent protective factors for SSI in this study. Adhesive incise drapes are utilized to separate the adjacent skin surfaces from the surgical wounds, preventing the migration of microorganisms [24]. In this study, there is one deficiency whether adhesive incise drapes are impregnated with antimicrobial materials. Moreover, two randomized controlled trials demonstrated that using incise drapes without antimicrobial properties had neither benefit nor harm for the prevention of SSI [25, 26]. Intriguingly, antimicrobial-impregnated adhesive incise drapes are still controversial in reducing the risk of SSI $[27,28]$. According to global guidelines for the prevention of SSI from World Health Organization (WHO) [29], it is unnecessary to use adhesive incise drapes with 
Table 3 Outcomes of included patients

\begin{tabular}{|c|c|c|c|c|}
\hline Variables & Total $(n=1046)$ & $\begin{array}{l}\text { Non-SSI group } \\
(n=972)\end{array}$ & $\begin{array}{l}\text { SSI group } \\
(n=74)\end{array}$ & $p$ value \\
\hline Length of hospital stay, day, median (IQR) & $16.0(12.0-21.0)$ & $15.0(12.0-21.0)$ & $22.5(17.0-33.0)$ & $<0.001$ \\
\hline Length of postoperative stay, day, median (IQR) & $9.0(7.0-12.0)$ & $9.0(7.0-12.0)$ & $15.0(11.0-24.0)$ & $<0.001$ \\
\hline 30-day mortality, n, (\%) & $6(0.6 \%)$ & $3(0.3 \%)$ & $3(4.1 \%)$ & 0.006 \\
\hline Medical cost, yuan, median (IQR) & $58,982(42972-79,262)$ & $57,827(42000-77,919)$ & $74,620(59078-107,612)$ & $<0.001$ \\
\hline Surgery to SSI time, day, median (IQR) & - & - & $5.5(3.0-9.0)$ & - \\
\hline SSI type, n, (\%) & - & - & & - \\
\hline Superficial incisional infection & - & - & $32(43.2 \%)$ & - \\
\hline Deep incisional infection & - & - & $18(24.3 \%)$ & - \\
\hline Organ-space infection & - & - & $24(32.4 \%)$ & - \\
\hline \multicolumn{5}{|l|}{ Pathogen, n, (\%) } \\
\hline Escherichia coli & - & - & $25(33.8 \%)$ & - \\
\hline Klebsiella pneumoniae & - & - & $4(5.4 \%)$ & - \\
\hline Enterococcus faecalis & - & - & $3(4.1 \%)$ & - \\
\hline Enterococcus faecium & - & - & $2(2.7 \%)$ & - \\
\hline Morganella morganii & - & - & $2(2.7 \%)$ & - \\
\hline Candida albicans & - & - & $2(2.7 \%)$ & - \\
\hline Pseudomonas aeruginosa & - & - & $1(1.4 \%)$ & - \\
\hline Acinetobacter baumanii & - & - & $1(1.4 \%)$ & - \\
\hline Staphylococcus aureus & - & - & $1(1.4 \%)$ & - \\
\hline Proteus mirabilis & - & - & $1(1.4 \%)$ & - \\
\hline Enterobacter cloacae & - & - & $1(1.4 \%)$ & - \\
\hline Staphylococcus epidermidis & - & - & $1(1.4 \%)$ & - \\
\hline Negative & - & - & $21(28.4 \%)$ & - \\
\hline None of germiculture & - & - & $17(23.0 \%)$ & - \\
\hline
\end{tabular}

or without antimicrobial properties for preventing SSI, but this is a conditional recommendation with low to very low quality of evidence. Taken together, we perhaps need more high quality randomized controlled trials to evaluate the influence of adhesive incise drapes on SSI.

Preoperative bowel preparation remains a controversial and intriguing issue around the world, with much debate regarding the use of MBP combined with or without OABP. Many studies demonstrated that MBP for colorectal surgery may not prevent SSI and improve outcome for patients [30,31]. In our study, results from univariate analysis showed the incidence of SSI in patients with MBP was significantly lower than those with no bowel preparation. However, the multivariate analysis revealed that the bowel preparation was not the independent risk factor of SSI in this study. Our previous study found that MBP combined with OABP significantly reduced SSI and minimized the readmission rates in contaminated and dirty types of colorectal surgery [32]. The global guideline from WHO suggests preoperative MBP combined with OABP should be used to reduce the risk of SSI in the patients receiving elective colorectal surgery [29]. Therefore, whether undergoing the bowel preparation and how to conduct the bowel preparation may depend on the different surgical wound class and surgical site. Furthermore, we found that 29 out of 331 patients $(8.8 \%)$ with no bowel preparation had contaminated or dirty wounds, and 13 out of 575 patients $(2.3 \%)$ receiving MBP had contaminated or dirty wounds in this study, which could account for the reason that patients receiving no bowel preparation had the higher SSI incidence. In addition, the most common method of bowel preparation was MBP alone (SSI rates: $4.5 \%$ ), only 42 patients received OABP (SSI rates: $14.3 \%$ ) and 98 patients underwent MBP with OABP (SSI rates: $5.1 \%$ ) in this study. Limited by the small number of cases (OABP with or without MBP), our study did not clearly reflect the difference among the bowel preparation of OABP with or without MBP. More cases should be collected to analyze the role of different bowel preparation in SSI after colorectal surgery. However, our study indicated that it was not very common to using oral antibiotics for the bowel preparation of colorectal surgery in China. 
Table 4 Univariate and multivariate logistic regression analysis of risk factors of SSI following colorectal surgery

\begin{tabular}{|c|c|c|c|c|}
\hline \multirow[t]{2}{*}{ Variables } & \multicolumn{2}{|c|}{ univariate } & \multicolumn{2}{|c|}{ multivariate } \\
\hline & $p$ value & OR $(95 \% \mathrm{Cl})$ & $p$ value & OR $(95 \% \mathrm{Cl})$ \\
\hline \multicolumn{5}{|l|}{ Hypertension } \\
\hline Yes & 0.007 & $1.975(1.207-3.232)$ & 0.024 & $1.903(1.088-3.327)$ \\
\hline \multicolumn{5}{|l|}{$\mathrm{No}^{\mathrm{a}}$} \\
\hline \multicolumn{5}{|l|}{ Tuberculosis } \\
\hline Yes & 0.017 & $8.972(1.475-54.559)$ & 0.083 & $7.880(0.762-81.492)$ \\
\hline \multicolumn{5}{|l|}{$\mathrm{No}^{\mathrm{a}}$} \\
\hline \multicolumn{5}{|l|}{ Albumin } \\
\hline \multicolumn{5}{|l|}{$\geq 3.5 \mathrm{~g} / \mathrm{dl}^{\mathrm{a}}$} \\
\hline$<3.5 \mathrm{~g} / \mathrm{dl}$ & $<0.001$ & $2.960(1.821-4.810)$ & 0.069 & $1.715(0.958-3.068)$ \\
\hline \multicolumn{5}{|l|}{ Blood glucose } \\
\hline \multicolumn{5}{|l|}{$80-150 \mathrm{mg} / \mathrm{dl}^{\mathrm{a}}$} \\
\hline$>150 \mathrm{mg} / \mathrm{dl}$ & 0.008 & $2.417(1.256-4.651)$ & 0.879 & 1.065(0.475-2.388) \\
\hline$<80 \mathrm{mg} / \mathrm{dl}$ & 0.637 & $1.166(0.617-2.200)$ & & \\
\hline \multicolumn{5}{|l|}{ Type of surgery } \\
\hline \multicolumn{5}{|l|}{ Rectal surgery ${ }^{a}$} \\
\hline Colon surgery & 0.002 & $2.419(1.370-4.269)$ & 0.154 & $0.631(0.335-1.188)$ \\
\hline \multicolumn{5}{|l|}{ Urgency of surgery } \\
\hline \multicolumn{5}{|l|}{ Elective $^{a}$} \\
\hline Emergency & $<0.001$ & $5.927(3.245-10.827)$ & 0.913 & 1.056(0.397-2.815) \\
\hline \multicolumn{5}{|l|}{ Timing of hair removal } \\
\hline \multicolumn{5}{|l|}{ None $^{a}$} \\
\hline Night before surgery & 0.009 & $0.477(0.272-0.834)$ & 0.342 & $0.711(0.352-1.442)$ \\
\hline Day of surgery & 0.311 & 0.698(0.348-1.399) & & \\
\hline \multicolumn{5}{|l|}{ Bowel preparation } \\
\hline \multicolumn{5}{|l|}{ None $^{a}$} \\
\hline MBP only & $<0.001$ & $0.376(0.223-0.634)$ & 0.185 & $0.650(0.344-1.229)$ \\
\hline OABP only & 0.554 & 1.324(0.523-3.355) & & \\
\hline $\mathrm{MBP}+\mathrm{OABP}$ & 0.083 & $0.427(0.163-1.119)$ & & \\
\hline \multicolumn{5}{|l|}{ Approach } \\
\hline \multicolumn{5}{|l|}{ Open $^{a}$} \\
\hline Laparoscopic or robotic & $<0.001$ & $0.259(0.157-0.429)$ & $<0.001$ & $0.363(0.200-0.659)$ \\
\hline \multicolumn{5}{|l|}{ Incisional protection } \\
\hline \multicolumn{5}{|l|}{ None $^{a}$} \\
\hline Wound edge protector & 0.545 & $0.833(0.460-1.507)$ & & \\
\hline Gauze & 0.368 & $0.676(0.288-1.585)$ & & \\
\hline Adhesive incise drapes & 0.031 & $0.472(0.239-0.932)$ & 0.018 & $0.400(0.187-0.855)$ \\
\hline \multicolumn{5}{|l|}{ Skin closure materials } \\
\hline Silk sutures ${ }^{a}$ & & & & \\
\hline Absorbable sutures & 0.060 & $0.524(0.268-1.027)$ & & \\
\hline Skin staples & 0.953 & 0.978(0.466-2.051) & & \\
\hline other & 0.053 & 2.496(0.988-6.307) & & \\
\hline Incisional wound irrigation & & & & \\
\hline None $^{a}$ & & & & \\
\hline
\end{tabular}


Table 4 Univariate and multivariate logistic regression analysis of risk factors of SSI following colorectal surgery (Continued)

\begin{tabular}{|c|c|c|c|c|}
\hline \multirow[t]{2}{*}{ Variables } & \multicolumn{2}{|c|}{ univariate } & \multicolumn{2}{|c|}{ multivariate } \\
\hline & $p$ value & OR $(95 \% \mathrm{CI})$ & $p$ value & OR $(95 \% \mathrm{Cl})$ \\
\hline Incisional wound irrigation & 0.111 & $0.465(0.181-1.193)$ & & \\
\hline Hydrogen peroxide solution & 0.317 & $0.500(0.129-1.942)$ & & \\
\hline Saline & 0.134 & $0.562(0.264-1.195)$ & & \\
\hline Other & 0.257 & $1.920(0.621-5.936)$ & & \\
\hline \multicolumn{5}{|l|}{ Colostomy/ileostomy } \\
\hline \multicolumn{5}{|l|}{$\mathrm{No}^{\mathrm{a}}$} \\
\hline Yes & $<0.001$ & $3.805(2.233-6.483)$ & 0.081 & $1.820(0.929-3.564)$ \\
\hline \multicolumn{5}{|l|}{ NNIS risk index } \\
\hline \multicolumn{5}{|l|}{0 or $1^{a}$} \\
\hline 2 or 3 & $<0.001$ & $6.481(3.655-11.493)$ & $<0.001$ & $3.840(1.926-7.658)$ \\
\hline
\end{tabular}

areference

There are many studies reporting that rectal operations had a higher risk than colectomies [33, 34]. Although rectal operations had a lower risk compared with colonic operations in this study, the multivariate analysis revealed that type of surgery was not the independent risk factor $(P=0.182)$ in Table 4 . Our data included the results of 2 years of cross-sectional studies (2018 and 2019). In 2018, the SSI incidence rate for colon surgery was $8.2 \%$ and the SSI incidence rate for rectal surgery was $3.8 \%$. In 2019, the SSI incidence rate for colon surgery and rectal surgery was 9.0 and $4.2 \%$, respectively. The results from 2 years of cross-sectional studies remain similar. Furthermore, 44 out of 641 patients (6.9\%) undergoing colon surgery had contaminated or dirty wounds, and 10 out of 405 patients $(2.5 \%)$ receiving rectal surgery had contaminated or dirty wounds in this study, which could lead to increased SSI incidence in colon surgery.

In this study, we carried out follow-up via standard telephone interview or review of the readmission records if patients were discharged from hospital after less than postoperative $30 \mathrm{~d}$. Data of patients who were lost to follow-up would not be included. Although there was withdraw bias, most patients have been followed up in this study. Therefore, our results are reliable. In addition, some limitations existed in the present study. Firstly, we only included patients receiving colorectal surgery from May 1 to June 30 of 2018 and the same time of 2019 . Some bias may be generated due to data collected during such a short period. Secondly, some details need to be improved, including the perioperative variation of blood pressure and different types of wound edge protectors and adhesive incise drapes.

\section{Conclusions}

Our study demonstrated that the nationwide incidence rate of SSI after colorectal surgery is $7.1 \%$. The prior diagnosis of hypertension, incisional protection (adhesive incise drapes), laparoscopic or robotic surgery, and NNIS risk index score of 2 or 3 may be associated with SSI occurrence after colorectal surgery. Considering drawbacks of this observational study, some other studies, such as randomized controlled trials, are needed to further identify risk factors for SSI following colorectal surgery. To further know nationwide SSI occurrence after colorectal surgery, the multicenter study to found more predictors for SSI in the following years should be conducted.

\section{Supplementary Information}

The online version contains supplementary material available at https://doi. org/10.1186/s12879-020-05567-6.

\section{Additional file 1.}

Additional file 2 .

\section{Abbreviations}

SSI: Surgical site infection; BMI: Body mass index; ASA score: American Society of Anesthesiologists physical status score; OABP: Oral antibiotics bowel preparation; MBP: Mechanical bowel preparation; The NNIS risk index: The national nosocomial infections surveillance risk index; SD: Standard deviation; IQR: Interquartile range

\section{Acknowledgments}

The authors are grateful to all patients and staff from the participating hospitals listed in Supplementary file 1 before data collection.

\section{Authors' contributions \\ Study conception and design: XFZ, XWW, JC and JR. Data collection: XFZ, ZWW, PW, SL, XJX, WM, GFW and GYL. Statistical analysis: XFZ, JC and XWW. Manuscript draft: XFZ and JR. All Authors commented the study and approved the final manuscript.}

\section{Funding}

National Major Scientific and Technological Special Project for "Significant New Drugs Development" (2017ZX09304005-002), Distinguished Scholars Foundation of Jiangsu Province (JCRCB2016006), Innovation Project of Military Medicine (16CXZ007), and General Project of Military Logistics Research (CLB19J025). The funders had neither role in the design of the 
study, collection, analysis, interpretation of data, nor in writing the manuscript.

\section{Availability of data and materials}

The datasets generated in the current study are not publicly available. However, the datasets used or analyzed in the current study will be available from the corresponding author on reasonable request.

\section{Ethics approval and consent to participate}

This study was approved by the ethics committee of Jinling hospital. Written informed consent was obtained from each study participant.

\section{Consent for publication}

The authors consent for publication of this manuscript.

\section{Competing interests}

The author states that there is no conflict of interest.

\section{Author details}

${ }^{1}$ Research Institute of General Surgery, Jinling Hospital, Nanjing Medical University, Nanjing 210002, People's Republic of China. ${ }^{2}$ Department of Surgery, The Second Affiliated Hospital, School of Medicine, Zhejiang University, Hangzhou 310009, People's Republic of China. ${ }^{3}$ Research Institute of General Surgery, Jinling Hospital, Medical School of Nanjing University, Nanjing 210002, People's Republic of China. ${ }^{4}$ Department of Emergency Surgery, The Affiliated Hospital of Qingdao University, Qingdao 266000, People's Republic of China. ${ }^{5}$ Department of Emergency Trauma Surgery, The People's Hospital of Xinjiang Uygur Autonomous Region, Urumqi 830001 People's Republic of China. 'Department of General Surgery, The First Affiliated Hospital of Xinjiang Medical University, Urumqi 830054, People's Republic of China. ${ }^{7}$ Department of Gastrointestinal Surgery, The People's Hospital of Guangxi Zhuang Autonomous Region, Nanning 530021, People's Republic of China. ${ }^{8}$ Department of Gastrointestinal Surgery, The People's Hospital of Hunan, Changsha 410005, People's Republic of China.

Received: 13 June 2020 Accepted: 30 October 2020

Published online: 12 November 2020

\section{References}

1. GlobalSurg C. Surgical site infection after gastrointestinal surgery in highincome, middle-income, and low-income countries: a prospective, international, multicentre cohort study. Lancet Infect Dis. 2018;18:516-25.

2. Ohno M, Shimada Y, Satoh M, Kojima Y, Sakamoto K, Hori S. Evaluation of economic burden of colonic surgical site infection at a Japanese hospital. J Hosp Infect. 2018;99:31-5.

3. Allegranzi B, Bischoff $\mathrm{P}$, de Jonge S, Kubilay NZ, Zayed B, Gomes SM, et al. New WHO recommendations on preoperative measures for surgical site infection prevention: an evidence-based global perspective. Lancet Infect Dis. 2016;16:e276-e87.

4. Elia-Guedea M, Cordoba-Diaz de Laspra E, Echazarreta-Gallego E, ValeroLazaro MI, Ramirez-Rodriguez JM, Aguilella-Diago V. Colorectal surgery and surgical site infection: is a change of attitude necessary? Int J Color Dis. 2017;32:967-74

5. Hennessey DB, Burke JP, Ni-Dhonochu T, Shields C, Winter DC, Mealy K. Risk factors for surgical site infection following colorectal resection: a multiinstitutional study. Int J Color Dis. 2016;31:267-71.

6. Du M, Liu B, Li M, Cao J, Liu D, Wang Z, et al. Multicenter surveillance study of surgical site infection and its risk factors in radical resection of colon or rectal carcinoma. BMC Infect Dis. 2019:19.

7. Ercole FF, Starling CE, Chianca TC, Carneiro M. Applicability of the national nosocomial infections surveillance system risk index for the prediction of surgical site infections: a review. Braz J Infect Dis. 2007;11:134-41.

8. Berrios-Torres SI, Umscheid CA, Bratzler DW, Leas B, Stone EC, Kelz RR, et al. Centers for Disease Control and Prevention guideline for the prevention of surgical site infection, 2017. JAMA Surg. 2017;152:784-91.

9. Pujol M, Limón E, López-Contreras J, Sallés M, Bella F, Gudiol F. Surveillance of surgical site infections in elective colorectal surgery. Results of the VINCat program (2007-2010). Enferm Infecc Microbiol Clin. 2012;30:20-5.

10. Pedroso-Fernandez Y, Aguirre-Jaime A, Ramos MJ, Hernandez M, Cuervo M, Bravo A, et al. Prediction of surgical site infection after colorectal surgery. Am J Infect Control. 2016:44:450-4.
11. Haleem A, Chiang HY, Vodela R, Behan A, Pottinger JM, Smucker J, et al. Risk factors for surgical site infections following adult spine operations. Infect Control Hosp Epidemiol. 2016;37:1458-67.

12. Morikane $K$, Honda $H$, Yamagishi T, Suzuki S, Aminaka M. Factors associated with surgical site infection in colorectal surgery: the Japan nosocomial infections surveillance. Infect Control Hosp Epidemiol. 2014;35:660-6.

13. DeHaas D, Aufderheide S, Gano J, Weigandt J, Ries J, Faust B. Colorectal surgical site infection reduction strategies. Am J Surg. 2016;212:175-7.

14. Krieger $Y$, Walfisch $A$, Sheiner E. Surgical site infection following cesarean deliveries: trends and risk factors. J Matern Fetal Neonatal Med. 2017;30:8-12.

15. Yang S, Liu G, Tang D, Cai. Evaluation intravenous drip Cephazolin prophylaxis of breast Cancer surgery site infection. J Craniofac Surg 2017;28: e527-ee31.

16. Yilmaz HO, Babazade R, Leung S, Zimmerman NM, Makarova N, Saasouh W, et al. Postoperative hypotension and surgical site infections after colorectal surgery. Anesth Analg. 2018;127:1129-36.

17. Wang Z, Chen J, Wang P, Jie Z, Jin W, Wang G, et al. Surgical site infection after gastrointestinal surgery in China: a multicenter prospective study. J Surg Res. 2019;240:206-18.

18. Li X, Nylander W, Smith T, Han S, Gunnar W. Risk factors and predictive model development of thirty-day post-operative surgical site infection in the veterans administration surgical population. Surg Infect. 2018;19:278-85.

19. Kiran RP, El-Gazzaz GH, Vogel JD, Remzi FH. Laparoscopic approach significantly reduces surgical site infections after colorectal surgery: data from national surgical quality improvement program. J Am Coll Surg. 2010; 211:232-8.

20. Howard DP, Datta G, Cunnick G, Gatzen C, Huang A. Surgical site infection rate is lower in laparoscopic than open colorectal surgery. Color Dis. 2010; 12:423-7.

21. Mihaljevic AL, Schirren R, Ozer M, Ottl S, Grun S, Michalski CW, et al. Multicenter double-blinded randomized controlled trial of standard abdominal wound edge protection with surgical dressings versus coverage with a sterile circular polyethylene drape for prevention of surgical site infections: a CHIR-net trial (BaFO; NCT01181206). Ann Surg. 2014;260:730-7 discussion 7-9.

22. Reid K, Pockney P, Draganic B, Smith SR. Barrier wound protection decreases surgical site infection in open elective colorectal surgery: a randomized clinical trial. Dis Colon Rectum. 2010:53:1374-80.

23. Sookhai S, Redmond HP, Deasy JM. Impervious wound-edge protector to reduce postoperative wound infection: a randomised, controlled trial. Lancet. 1999;353:1585.

24. French ML, Eitzen HE, Ritter MA. The plastic surgical adhesive drape: an evaluation of its efficacy as a microbial barrier. Ann Surg. 1976;184:46-50.

25. Ward HR, Jennings OG, Potgieter P, Lombard CJ. Do plastic adhesive drapes prevent post caesarean wound infection? J Hosp Infect. 2001;47:230-4.

26. Chiu KY, Lau SK, Fung B, Ng KH, Chow SP. Plastic adhesive drapes and wound infection after hip fracture surgery. Aust N Z J Surg. 1993;63:798-801.

27. Yoshimura Y, Kubo S, Hirohashi K, Ogawa M, Morimoto K, Shirata K, et al. Plastic iodophor drape during liver surgery operative use of the iodophorimpregnated adhesive drape to prevent wound infection during high risk surgery. World J Surg. 2003;27:685-8.

28. Al-Qahtani SM, Al-Amoudi HM, Al-Jehani S, Ashour AS, Abd-Hammad MR, Tawfik OR, et al. Post-appendectomy surgical site infection rate after using an antimicrobial film incise drape: a prospective study. Surg Infect. 2015;16: $155-8$.

29. World Health Organization. Global Guidelines for the prevention of surgical site infection. In: Book World Health Organization. Global Guidelines for the prevention of surgical site infection. Geneva: World Health Organization; 2018.

30. Guenaga KF, Matos D, Wille-Jorgensen P. Mechanical bowel preparation for elective colorectal surgery. Cochrane Database Syst Rev. 2011;(9):CD001544.

31. Koskenvuo L, Lehtonen T, Koskensalo S, Rasilainen S, Klintrup K, Ehrlich A, et al. Mechanical and oral antibiotic bowel preparation versus no bowel preparation for elective colectomy (MOBILE): a multicentre, randomised, parallel, single-blinded trial. Lancet. 2019;394:840-8.

32. Anjum N, Ren J, Wang G, Li G, Wu X, Dong H, et al. A randomized control trial of preoperative Oral antibiotics as adjunct therapy to systemic antibiotics for preventing surgical site infection in clean contaminated, contaminated, and dirty type of colorectal surgeries. Dis Colon Rectum. 2017:60:1291-8 
33. Murray AC, Pasam R, Estrada D, Kiran RP. Risk of surgical site infection varies based on location of disease and segment of colorectal resection for Cancer. Dis Colon Rectum. 2016;59:493-500.

34. Ghuman A, Kasteel N, Brown CJ, et al. Surgical site infection in elective colonic and rectal resections: effect of oral antibiotics and mechanicalbowel preparation compared with mechanical bowel preparation only [published online ahead of print, 2020 May 22]. Colorectal Dis. 2020.

\section{Publisher's Note}

Springer Nature remains neutral with regard to jurisdictional claims in published maps and institutional affiliations.

Ready to submit your research? Choose BMC and benefit from:

- fast, convenient online submission

- thorough peer review by experienced researchers in your field

- rapid publication on acceptance

- support for research data, including large and complex data types

- gold Open Access which fosters wider collaboration and increased citations

- maximum visibility for your research: over $100 \mathrm{M}$ website views per year

At $\mathrm{BMC}$, research is always in progress.

Learn more biomedcentral.com/submissions 